\title{
Efficient Ho:LuLiF 4 laser in-band pumped by a Tm fiber laser
}

\author{
J. W. Kim, ${ }^{1, *}$ J. I. Mackenzie, ${ }^{1}$ D. Parisi, ${ }^{2}$ S. Veronesi, ${ }^{2}$ M. Tonelli, ${ }^{2}$ and W. A. Clarkson ${ }^{1}$ \\ ${ }^{1}$ Optoelectronics Research Centre, University of Southampton, Southampton, SO 17 IBJ, UK \\ ${ }^{2}$ National Enterprise for Nano Science and Technology-INFM - CNR, Dipartimento di Fisica Università di Pisa, Largo B. Pontecorvo 3, 56127 \\ Pisa, Italy \\ *e-mail:jwk@orc.soton.ac.uk
}

\begin{abstract}
A Ho:LuLiF 4 laser with an output power of $5.4 \mathrm{~W}$ and slope efficiency of $76 \%$ at $2.1 \mu \mathrm{m}$ in-band pumped by a Tm fiber laser at $1.94 \mu \mathrm{m}$ is reported. Strategies for further increasing output power are considered.

(C) 2009 Optical Society of America

OCIS codes: (140.0140) Lasers and laser optics; (140.3070) infrared and far-infrared laser; (140.3580) Lasers, solid-state
\end{abstract}

Efficient solid-state lasers in the eyesafe 2-micron regime are required for a number of applications including remote sensing, coherent LIDAR, medicine, and as a pump source for generating coherent mid infrared radiation via nonlinear frequency conversion. $\mathrm{LuLiF}_{4}$ (LLF) [1,2], like its isomorphs $\mathrm{YLiF}_{4}$ and $\mathrm{GdLiF}_{4}$, has the advantages of strong spectral anisotropy for polarized absorption and emission, a long upper-level lifetime of nearly $15 \mathrm{~ms}$ for energy storage in the gain medium and a negative thermal dependence of refractive index (dn/dT), which partially compensates the positive bulging of the end faces of the laser rod due to the positive thermal expansion coefficients, leading to weak overall thermal lensing. LLF also benefits from a closer match in its thermal expansion coefficient and conductivity along each of the crystal axes with respect to its isomorphs.

Here we report what we believe to be the first demonstration of an efficient continuous-wave Ho:LLF laser at $2.1 \mu \mathrm{m}$, in-band pumped by a linearly-polarized Tm fiber laser at $1.94 \mu \mathrm{m}$. The Ho:LLF laser was successfully operated with an output power of $5.4 \mathrm{~W}$ at $2.1 \mu \mathrm{m}$ for an absorbed pump power of $8 \mathrm{~W}$, corresponding to a slope efficiency of $76 \%$. Laser performance under different operating conditions together with the prospects for further power and energy scaling will be discussed.

The Ho-doped LLF crystal used in our experiments was grown by a Czochralski technique with a Ho doping concentration of approximately 0.25 at.\%. The crystal sample used in our laser had dimensions of $(5 \times 5 \times 40) \mathrm{mm}^{3}$, with the crystallographic c-axis orthogonal to the longest edge and was prepared from the a crack-free Ho:LLF boule of $\sim 10 \mathrm{~mm}$ in diameter and $\sim 70 \mathrm{~mm}$ in length. The Ho:LLF laser configuration used in our experiments is shown in Fig. 1. The Tm fiber pump laser was constructed in-house and comprised a $\sim 4 \mathrm{~m}$ length of a commercially available Tm-doped polarization-maintaining (PM) fiber with a core diameter of $25 \mu \mathrm{m}(0.1 \mathrm{NA})$, and a cladding diameter of $400 \mu \mathrm{m}(0.4 \mathrm{NA})$. The fiber laser's operating wavelength was selected to coincide with the absorption peak in Ho:LLF at $1937 \mathrm{~nm}$ using an external feedback cavity arrangement comprising a collimating lens (f $=100$ $\mathrm{mm})$, half-wave plate, polarizer and a diffraction grating (600 lines $/ \mathrm{mm})$ in the Littrow configuration. Pump light at $795 \mathrm{~nm}$ was provided by two spatially- and polarization-multiplexed diode-stacks. The Tm fiber laser output beam was collimated and an additional half-wave plate and polarizer used to improve its polarization extinction ratio while providing the ability to align the electric field to the c-axis of the Ho:LLF crystal, which corresponds to the strongest available absorption line.

A simple three-mirror folded resonator was employed comprising a plane pump input-coupler (IC) with high reflectivity (> 99.8\%) around the lasing wavelength $(2050-2150 \mathrm{~nm})$ and high transmission $(>95 \%)$ at the pump wavelength $(1937 \mathrm{~nm})$, a convex lens $(\mathrm{f}=100 \mathrm{~mm})$, one fold mirror $(\mathrm{DM})$ with the same reflection/transmission characteristics as the IC allowing unabsorbed pump power to be monitored, and a plane output coupler. Laser performance for a range of output coupler mirror transmissions $\left(\mathrm{T}_{\mathrm{oc}}\right)$ of $3 \%, 10 \%, 20 \%$ and $37 \%$ was investigated. Both end faces of the Ho:LLF crystal were antireflection coated for the 1.9 to $2.1 \mu \mathrm{m}$ wavelength range, covering both pump and lasing wavelengths. For room temperature operation, the Ho:LLF crystal was mounted in a watercooled copper heat-sink maintained at a temperature of $17^{\circ} \mathrm{C}$ and positioned in close proximity to the plane mirror (IC). The physical length of the resonator was $\sim 300 \mathrm{~mm}$ resulting in a calculated TEM $_{00}$ waist radius of $\sim 200 \mu \mathrm{m}$ and, hence, the pump beam from the Tm fiber laser was conditioned to have a similar beam waist size in the Ho:LLF crystal.

Figure 2 shows the output power as a function of absorbed pump power. For output coupler transmissions of $3 \%, 10 \%$ and $20 \%$, the laser operated at a wavelength of $2066 \mathrm{~nm}$ and the slope efficiency increases with output coupler transmission due to the higher proportion of useful output coupling to loss in agreement with expectations. The laser with the $20 \%$ transmitting output coupler produced $5.1 \mathrm{~W}$ of output for $8.0 \mathrm{~W}$ of absorbed pump with a corresponding slope efficiency of $70 \%$ with respect to absorbed power. Threshold pump power was measured to be 
$420 \mathrm{~mW}, 440 \mathrm{~mW}$ and $470 \mathrm{~mW}$ for $3 \%, 10 \%$, and $20 \% \mathrm{~T}_{\mathrm{oc}}$ output coupling mirrors respectively. This indicates that a large fraction of the excitation density required to reach threshold can be attributed to the three-level character of the transition. Using the $37 \% \mathrm{~T}_{\mathrm{oc}}$ output coupler shifts the operating wavelength of the Ho:LLF laser to $2053 \mathrm{~nm}$ since the effective gain at this wavelength exceeds that at $2066 \mathrm{~nm}$ due to the higher excitation density required to reach threshold. For this output coupler, the maximum output power was $5.4 \mathrm{~W}$ for $8.0 \mathrm{~W}$ of absorbed pump power and the corresponding slope efficiency was 76\%. Threshold pump power was measured to be only $550 \mathrm{~mW}$. The beam propagation factor $\left(\mathrm{M}^{2}\right)$ was measured to be $\sim 1.1$ at the maximum output power confirming near diffractionlimited $\mathrm{TEM}_{\mathrm{oo}}$ operation.

Scaling to higher powers should be possible through optimizing doping concentration, pump absorption efficiency and operating conditions. Operation of Ho:LLF at cryogenic temperatures looks particularly promising and should yield a dramatic improvement in performance at high pump powers by virtue of offering more than 100fold improvement in thermo-optic figures-of-merit as well as dramatically reduced three-level character compared to room temperature. The results of a preliminary studies on cryogenically-cooled operation of Ho:LLF laser will also be presented.

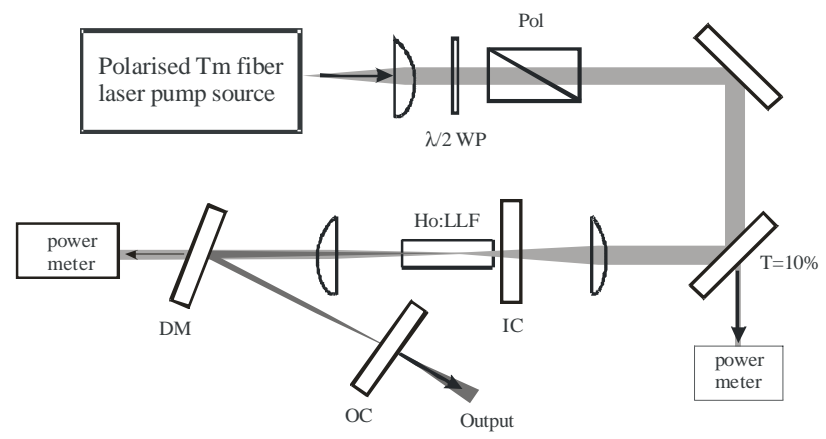

Fig. 1. Schematic diagram of the Ho:LLF resonator.

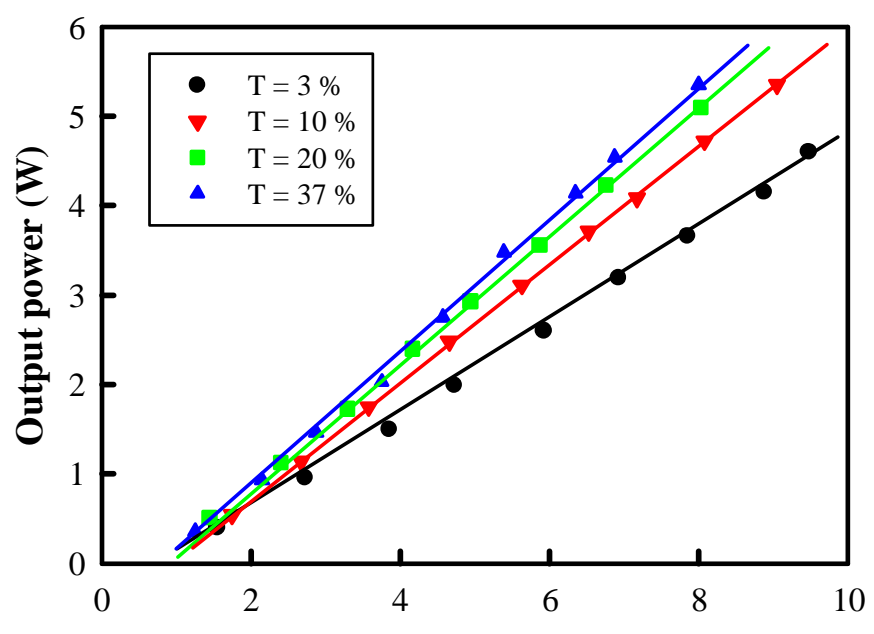

Absorbed pump power (W)

Fig. 2. Output power versus absorbed pump power for the Ho:LLF laser using output couplers with transmissions of 3\%, 10\%, $20 \%$ and $37 \%$.

[1] F. Cornacchia, A. Toncelli, M. Tonelli, “2- $\mu \mathrm{m}$ lasers with fluoride crystals:Research and development," Prog. in Quantum Electron 33, 61109 (2009).

[2] B. M. Walsh, N. P. Barnes, M. Petros, J. Yu and U. N. Singh, "Spectroscopy and modeling of solid state lanthanide lasers: Application to trivalent $\mathrm{Tm}^{3+}$ and $\mathrm{Ho}^{3+}$ in $\mathrm{YLiF}^{4}$ and $\mathrm{LuLiF}^{4}$," J. Appl. Phys. 95, 3255-3271 (2004). 